\title{
Sleep-disordered breathing and genetic findings in children with Prader-Willi syndrome in China
}

\author{
Aizhen $\mathrm{Lu}^{1}$, Feihong $\mathrm{Luo}^{2}$, Chengjun Sun ${ }^{2}$, Xiaobo Zhang ${ }^{1}$, Libo Wang ${ }^{1}$, Wei Lu ${ }^{2}$ \\ ${ }^{1}$ Department of Respiratory, ${ }^{2}$ Department of Endocrinology and Inherited Metabolic Diseases, Children's Hospital of Fudan University, Shanghai, \\ China \\ Contributions: (I) Conception and design: A Lu, W Lu; (II) Administrative support: None; (III) Provision of study materials or patients: None; (IV) \\ Collection and assembly of data: A Lu, X Zhang, L Wang, C Sun; (V) Data analysis and interpretation: A Lu, X Zhang, L Wang, F Luo, W Lu; (VI) \\ Manuscript writing: All authors; (VII) Final approval of manuscript: All authors. \\ Correspondence to: Dr. Wei Lu. Department of Endocrinology and Inherited Metabolic Diseases, Children's Hospital of Fudan University, No 399, \\ Wanyuan Road, Minhang District, Shanghai, China. Email: wei_lu77@163.com.
}

Background: Sleep-related breathing disorders are common in individuals with Prader-Willi syndrome (PWS), and can include hypersomnolence and obstructive sleep apnea, as well as central sleep breathing abnormalities that are present from infancy. Here we describe the sleep-disordered breathing (SDB) and genetic findings in patients with PWS in China.

Methods: In all, 48 patients confirmed by genetic tests were enrolled, 32 were under 2 years of age and 16 were older children. There were 37 (77.1\%) patients with paternal 15q11-13 deletions, 11 (22.9\%) patients with maternal uniparental disomy (mUPD), and no patients with imprinting defect (ID).

Results: Compared with infants, a significantly higher proportion of older children with PWS were overweight or obese (15/16 children vs. 4/32 infants) and children had a higher serum level of free thyroxine (FT4) $(0.9 \pm 0.2$ vs. $0.7 \pm 0.7)$ and thyroxine (T4) $(9.0 \pm 2.5$ vs. $7.5 \pm 1.7)$. Age was correlated significantly with body mass index (BMI), T4, and FT4 (r=0.626, P=0.000; r=0.426, respectively). Overall, 42 of 48 (87.5\%) patients had sleep apnea on polysomnography (PSG). Infants, when compared with older children, were more likely to experience central sleep apnea $(71.8 \%$ vs. $25 \%)$. In infants, there were no significant differences in the prevalence of SDB between the deletion group and the mUPD group.

Conclusions: Being overweight or obese was more common in older children with PWS. Compared with infants, a higher proportion children were overweight or obese and had higher serum levels of FT4 and T4. The prevalence of SDB was high in those with PWS, and central sleep apnea was found to be prevalent in infants. The pattern of SDB in infants with PWS was not significantly associated with the genotypes.

Keywords: Prader-Willi syndrome (PWS); sleep-disordered breathing (SDB); polysomnography (PSG); sleep apnea; genotype

Submitted May 15, 2020. Accepted for publication Jul 23, 2020.

doi: 10.21037/atm-20-4475

View this article at: http://dx.doi.org/10.21037/atm-20-4475

\section{Introduction}

Prader-Willi syndrome (PWS) is a complex genetic disorder that occurs in 1 in 10,000 to 1 in 25,000 live births (1). Genomic imprinting errors in the 15q11-q13 chromosome region, which can occur during gametogenesis in both sexes, are the cause of PWS and give rise to the loss of expression in paternal genes (2-6). PWS is characterized by severe hypotonia, poor feeding, hypogonadism, hyperphagia, obesity, short stature, sleep-disordered breathing (SDB), and multiple endocrine abnormalities $(1,7)$. Treatment with recombinant human growth hormone (rhGH) starting in infancy has improved timing of developmental milestones, growth, obesity, and cognition. Investigation into neurologic, metabolic and hormonal drivers of hyperphagia 
and obesity has revealed new insights and clarified the underlying pathophysiology (8). Additional studies continue to elucidate the hormonal deficiencies seen in the syndrome, allowing for improvements in clinical care. The aim of this study was to describe SDB and genetic findings in patients with PWS in China. Understanding the nature and underlying causes of hypotonia, hyperphagia, hormonal dysfunction or SDB in this syndrome is necessary to guide current management and future research directions. We present the following article in accordance with the STROBE reporting checklist (available at http://dx.doi. org/10.21037/atm-20-4475).

\section{Methods}

\section{Patients}

This retrospective study evaluated data of pediatric patients with PWS who were admitted to Children's Hospital of Fudan University from January 2015 to December 2017. PWS was confirmed by genetic tests. Children were separated into two groups: the infants group ( $\leq 2$ years old) and the older children group ( $>2$ years old). The exclusion criteria were the following: (I) patients already beginning growth hormone $(\mathrm{GH})$ treatment, or (II) patients already treated for SDB. The study was approved by the Medical Ethics Committee of Children's Hospital of Fudan University. This study was conducted in accordance with the Declaration of Helsinki (as revised in 2013). Written consent was acquired from both parents of each patient before study participation.

\section{Genetic analysis}

Genomic DNA extracted from peripheral blood was used for methylation-specific polymerase chain reaction (MSPCR) to determine subjects' methylation status. Subjects with abnormal methylation pattern were subjected to further testing for subtypes of genetic defects. We used methylation-specific multiplex ligation-dependent probe amplification (MS-MLPA) to distinguish deletion in 15q11-q13 from maternal uniparental disomy (mUPD) and imprinting defect (ID), followed by short tandem repeat linkage analysis to distinguish UPD from ID.

\section{Demographic characteristics}

Demographic characteristics (sex, age, height, and weight) were collected. Body mass index (BMI) was calculated as follows: weight $(\mathrm{kg}) /$ height $\left(\mathrm{m}^{2}\right)$. BMI z-score was calculated using age- and sex-specific growth curves according to the World Health Organization (WHO) who defined overweight status as a BMI z-score between 1 and 2 and obesity as a BMI z-score $\geq 2$ (9). All recruited subjects were examined by nasopharyngeal computed tomography (CT) scan for upper airway obstruction before overnight polysomnography (PSG). The size of the adenoid in patients with nasopharyngeal abnormalities was assessed using nasal endoscopy. We conducted clinical workup in all patients using a standardized procedure with focus on common PWS complications (epilepsy, scoliosis, cardiovascular diseases).

\section{Laboratory tests}

Blood samples were drawn at approximately 8:00 a.m. after an overnight fast. Serum levels of cholesterol (CHOL), triglyceride (TG), high-density lipoprotein (HDL), low-density lipoprotein (LDL), blood glucose (GLU), glycosylated hemoglobin (HbA1C), insulin-like growth factor 1 (IGF-1), insulin growth factor binding protein-3 (IGFBP-3), cortisol, adrenocorticotropic hormone (ACTH), thyroxine (T4), triiodothyronine (T3), thyroid stimulating hormone (TSH), free T3 (FT3), and free T4 (FT4) were determined using local routine laboratory assays.

\section{PSG evaluation}

Nocturnal sleep ( $\geq 6$ hours) PSG (Compumedics Ltd., Australia) was performed to record the following parameters: electroencephalogram, electrooculogram, submental and limb electromyogram, electrocardiography, oronasal airflow, thoracic and abdominal movements, percutaneous oxygen saturation $(\mathrm{SpO} 2)$ and body positions. Respiratory events including obstructive sleep apnea (OSA) and central sleep apnea (CSA) were scored based on the recommendation from The American Academy of Sleep Medicine's (AASM) Manual for the Scoring of Sleep and Associated Events. We used the following definitions for obstructive apnea index (OAI) and central apnea index (CAI): an average OAI or CAI of more than 1 event per hour with a minimum $\mathrm{SpO} 2$ nadir of $92 \%$ during sleep was considered abnormal (10).

\section{Statistical analysis}

Statistical analyses were carried out with SPSS (version 
Table 1 Demographic and anthropometric data in children with Prader-Willi syndrome

\begin{tabular}{|c|c|c|c|c|}
\hline Variable & All patients $(n=48)$ & Age $\leq 2$ years $(n=32)$ & Age $>2$ years $(n=16)$ & $P$ value \\
\hline Female & 16 & 11 & 5 & 0.829 \\
\hline BMI z-score & $0.8 \pm 3.2$ & $-1.01 \pm 1.9$ & $4.2 \pm 2.4$ & $0.000^{\star \star}$ \\
\hline Height/length z-score & $-0.9 \pm 2.0$ & $-1.1 \pm 2.3$ & $-0.6 \pm 1.4$ & 0.406 \\
\hline Epilepsy & 5 & 4 & 1 & 0.652 \\
\hline Scoliosis & 7 & 4 & 3 & 0.672 \\
\hline Cardiovascular diseases & 5 & 3 & 2 & 0.546 \\
\hline Adenoid hypertrophy & 5 & 2 & 3 & 0.316 \\
\hline
\end{tabular}

**, $\mathrm{P}<0.01$. BMI, body mass index.

18.0, SPSS Inc.). Categorical variables were compared between groups using Chi-square test. Continuous variables were presented as mean \pm standard deviation or medians with ranges, and were analyzed using $t$-test or Wilcoxon rank-sum test. The association between parameters in patients with PWS was performed by multivariate logistic regression. $\mathrm{P}$ values $<0.05$ were considered statistically significant.

\section{Results}

\section{Genotypes}

We enrolled 48 children with PWS. There were 32 patients in the infant group and 16 patients in the older children group. Genetic studies showed paternal 15q11-13 deletions in $37(77 \%)$ patients and mUPD in $11(23 \%)$ patients. No ID was found in our study. Among 11 patients with mUPD, 10 were infants. There was no significant difference in genotypes between the infants and older children group.

\section{Demographical and anthropometric characteristics}

The median age of the 48 patients with PWS was 16.8 months (range, 3-188 months), BMI z-score was $0.8 \pm 3.2$, and height $z$-score was $-0.9 \pm 2.0$. The median age in the infant and older children groups was 9.1 months (range, 3-23.5 months) and 73.9 months (range, 26.3-188 months) respectively, BMI z-score was $-1.0 \pm 1.9$ and $4.2 \pm 2.3$ respectively, and the height $z$-score was $-1.1 \pm 2.3$ and $-0.6 \pm 1.4$ respectively. There was a significantly higher proportion of overweight or obese children (15/16) with PWS when compared with infants $(4 / 32 ; \mathrm{P}=0.000)$. There were 5 patients who had epilepsy, 7 patients with scoliosis, 5 patients with cardiovascular diseases, and 5 patients with adenoid hypertrophy. There were no significant differences in the incidences of complications between the two groups (Table 1).

\section{Laboratory tests}

There were significant differences between the infant group and the older children group in serum levels of IGF-1 (31.5 \pm 14.7 vs. $126.7 \pm 65.3 \mathrm{ng} / \mathrm{mL}, \mathrm{P}=0.001)$, FT4 $(0.7 \pm 0.2$ vs. $0.9 \pm 0.2, \mathrm{P}=0.01)$ and $\mathrm{T} 4(7.5 \pm 1.7$ vs. $9.0 \pm 2.5, \mathrm{P}=0.02)$. The other results of laboratory tests were not significantly different between infants and children. In infants, there were no significant differences in lab results between the deletion group and the mUPD group.

Table 2 shows the correlation coefficients between age, BMI z-score, IGF-1, T4, and FT4 within the total group of PWS patients. Age was correlated significantly with BMI $\mathrm{z}$-score, IGF-1, T4, and FT4 ( $\mathrm{r}=0.626, \mathrm{P}=0.000 ; \mathrm{r}=0.610$, $\mathrm{P}=0.000 ; \mathrm{r}=0.426, \mathrm{P}=0.003 ; \mathrm{r}=0.435, \mathrm{P}=0.003$; respectively). There was also significant correlation between $\mathrm{BMI}$ z-score and IGF-1, T4, FT4 (r=0.680, P=0.000; r=0.413, P=0.007; $\mathrm{r}=0.381, \mathrm{P}=0.013$; respectively). However, there were no significant correlations between IGF-1 and T4 or FT4.

\section{PSG results}

SDB was common in both infants and older children with 
Table 2 Correlation coefficients between age, BMI Z score, IGF-1, T4, FT4, and AHI in children with Prader-Willi syndrome

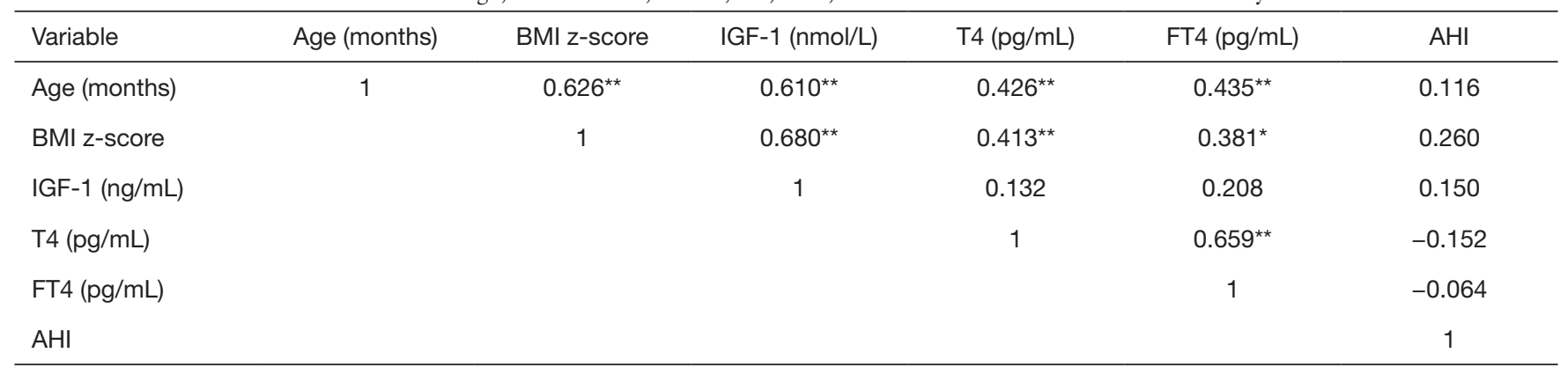

*, Significant correlation, $\mathrm{P}<0.05$; ${ }^{*}, \mathrm{P}<0.01$. BMI, body mass index; IGF-1, insulin-like growth factor-1; T4, thyroxine; FT4, free thyroxine; $\mathrm{AHI}$, apnea-hypopnea index.

Table 3 Polysomnography (PSG) in children with Prader-Willi syndrome

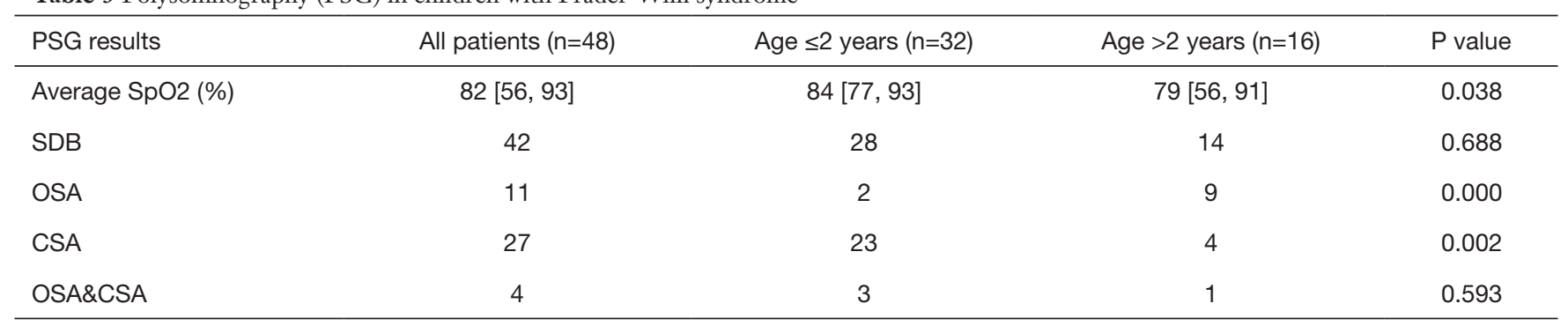

PSG, polysomnography; SpO2, peripheral capillary oxygen saturation; SDB, sleep-disordered breathing; OSA, obstructive sleep apnea; CSA, central sleep apnea.

Table 4 Polysomnography study in infants with Prader-Willi syndrome

\begin{tabular}{lccc}
\hline Variable & Deletion $(n=22)$ & $\operatorname{mUPD}(n=10)$ & $P$ value \\
\hline OSA & 2 & 0 & 0.466 \\
CSA & 15 & 8 & 0.405 \\
OSA and CSA & 2 & 1 & 0.69 \\
\hline
\end{tabular}

mUPD, maternal uniparental disomy; OSA, obstructive sleep apnea; CSA, central sleep apnea.

PWS (Table 3). Overall, 42/48 (87.5\%) patients had sleep apnea on the PSG. Specifically, 11 patients had OSA, 27 patients had CSA, and 4 patients had both OSA and CSA. No significant differences in the prevalence of sleep apnea were observed between infants and older children groups (Table 3, overall $\mathrm{P}=0.688$ ). Compared with older children, infants were more likely to have CSA (23 vs. 4; $\mathrm{P}=0.002)$, and less likely to have OSA (0 vs. 9; $\mathrm{P}=0.000$ ). The median $\mathrm{SpO} 2$ nadir was significantly lower in older children group [79\% (range 56, 91) vs. 84\% (range 77, 93); $\mathrm{P}=0.038]$. In infants, there were no significant differences in the prevalence of SDB between the deletion group and the mUPD group (Table 4). In addition, there was no correlation between the apnea-hypopnea index and age, BMI z-score, IGF-1, T4, or FT4 (Table 2).

\section{Discussion}

In this study, we found that overweight status or obesity was more common in older children with PWS. Serum level of FT4 and T4 were significantly higher in children than in infants. Age was correlated significantly with BMI, FT4, and T4. The correlation between age and BMI was consistent with the reported results $(7,11)$. These results indicate that the clinical features of PWS can change over time and remind us of the importance of early diagnosis and intervention. Although PWS is characterized by obesity with increased serum lipid profile, we found glucose homeostasis and lipid profiles seemed to be within the normal range in our patients, which was inconsistent with previous reports (12-15). We also found that serum level of IGF-1 was higher in children than in infants, and age was correlated significantly with IGF-1, which was consistent 
with the pattern that serum IGF-1 levels increase with age in childhood (16). Because of the small number of cases we studied, we need to collect more cases to study the metabolic characteristics of PWS patients at different ages.

Hypothalamic dysfunction is common in PWS, and therefore patients are at increased risk for central hypothyroidism. Previous studies have shown varying prevalence of hypothyroidism in PWS from as common as high as $20-30 \%$ to as low as $2-4 \%(17-19)$. One study examining thyroid function over the first 2 years of life reported a higher rate of central hypothyroidism in patients with PWS having low free or total T4 levels, with normal TSH. Therefore, TSH alone may not be enough to rule out hypothyroidism in neonates with PWS. In this study, the correlation of age and T4/FT4 in PWS showed that thyroid function was improved with age in PWS children. Since the relatively low level of T4/FT4 may be associated with the clinical manifestation in infants, it is suggested to screen for hypothyroidism within the first 3 months of life and then yearly thereafter, even if the newborn's screening results are normal, especially if on GH therapy. In this study, only 1 patient, who was 1 year old, was diagnosed with hypothyroidism based on low total T4 and FT4 in the presence of normal TSH.

The incidence of different genetic mechanisms resulting in PWS varies between studies $(11,20-24)$, and it is difficult to ascertain whether these differences were indeed due to ethnic differences or mere sampling bias. Our study showed that there was no significant difference in BMI, laboratory tests, and incidence of SDB between the deletion and mUPD groups in infants. However, in adults with PWS, deletion was reported to associated with increased BMI (25). Interestingly, in patients previously treated with GH, BMI was not different between the mUPD and deletion groups (25), and this suggests that genotype may be associated with $\mathrm{GH}$ secretion.

The prevalence of SDB in PWS in this study (87.5\%) was higher than that in previous studies (20-79.9\%) (4,26-29). Different criteria of SDB diagnosis maybe the main reason for this discrepancy in prevalence. Our criteria was not overly conservative; therefore, our results suggest the true incidence of SDB in PWS may be under evaluated and underappreciated in clinical practice. The basis of the sleep abnormalities in PWS include brain developmental abnormalities, craniofacial dysmorphia, hypotonia, obesity, and chest wall deformities (29). Traditionally, PWS has been associated with OSA (30-32), and BMI plays a significant role in increasing OSA risk in PWS children (29). rhGH therapy can theoretically lead to lymphoid tissue growth at the initial stage in PWS patients due to increased IGF-I effects, which can exacerbate obstructive apnea and may even lead to sudden death. Given this, patients and parents must be fully informed about the potential association between rhGH therapy and unexpected death during the pretreatment consenting process especially in patients with obstructive apnea. In this study, $56.3 \%$ of older children had OSA. This suggests that the number of infants (71.8\%) with CSA was high in our study, which is in line with the findings of other studies $(33,34)$. The etiology underlying this higher prevalence of CSA in young children with PWS is still unknown, but it may be related to delays in maturation of the central ventilatory control centers, or to an abnormal apneic threshold which is defined as the arterial partial pressure of $\mathrm{CO}_{2}$ below which the drive to breathe is lost (35). One study reported that treatment with GH for 6-9 months improved resting ventilation and ventilatory response to $\mathrm{CO}_{2}$ in subjects with PWS (15). Another study reported that GH intervention improved body composition and resting energy expenditure and may contribute to better sleep quality. These studies suggest that GH treatment may improve CSA in infants with PWS $(36,37)$. Since PWS patients have a high incidence of both central apnea and obstructive apnea, PSG should be performed before starting therapy.

Some limitations in this study should be addressed. First, this study was a retrospective study, and we could not compare SDB in patients with PWS to that in controls. Second, our sample size was relatively small; the sample size was thus not equal between the infant and older children group which might have introduced some bias. Third, this study focused on patients from one single center. In addition, we only identified one patient with mUDP in the older children group, and perhaps the difference of phenotypes between different genotypes in Chinese children with PWS requires a larger future study.

In summary, overweight status or obesity was more common in older children with PWS in China and other countries and regions. Compared with infants, children had a higher serum level of FT4 and T4. Age was correlated significantly with BMI, T4, and FT4. We thus suggest screening PWS patients for hypothyroidism within the first few months or years even if the newborn's screening results are normal. The prevalence of SDB was high in PWS patients, and central sleep apnea was found to be prevalent in infants with PWS, suggesting that PSG should be performed early to screen for SDB in PWS. The association 


\section{Page 6 of 7}

of central sleep apnea and level of FT4 and T4 in infants should be explored further.

\section{Acknowledgments}

Funding: The research was supported by Children's Hospital of Fudan University (No. EK112520180204) and Shanghai Science and Technology Committee (No. 17411960700).

\section{Footnote}

Reporting Checklist: The authors have completed the STROBE reporting checklist. Available at http://dx.doi. org/10.21037/atm-20-4475

Data Sharing Statement: Available at http://dx.doi. org/10.21037/atm-20-4475

Conflicts of Interest: All authors have completed the ICMJE uniform disclosure form (available at http://dx.doi. org/10.21037/atm-20-4475). The authors have no conflicts of interest to declare.

Ethical Statement: The authors are accountable for all aspects of the work in ensuring that questions related to the accuracy or integrity of any part of the work are appropriately investigated and resolved. The study was approved by the Medical Ethics Committee of Children's Hospital of Fudan University. This study was conducted in accordance with the Declaration of Helsinki (as revised in 2013). Written consent was acquired from both parents of each patient before study participation.

Open Access Statement: This is an Open Access article distributed in accordance with the Creative Commons Attribution-NonCommercial-NoDerivs 4.0 International License (CC BY-NC-ND 4.0), which permits the noncommercial replication and distribution of the article with the strict proviso that no changes or edits are made and the original work is properly cited (including links to both the formal publication through the relevant DOI and the license). See: https://creativecommons.org/licenses/by-nc-nd/4.0/.

\section{References}

1. Cassidy SB, Schwartz S, Miller JL, et al. Prader-Willi syndrome. Genet Med 2012;14:10-26.

2. Nixon GM, Brouillette RT. Sleep and breathing in Prader-
Lu et al. Sleep-disordered breathing in Prader-Willi syndrome

Willi syndrome. Pediatr Pulmonol 2002;34:209-17.

3. Bruni O, Verrillo E, Novelli L, et al. Prader-Willi syndrome: sorting out the relationships between obesity, hypersomnia, and sleep apnea. Curr Opin Pulm Med 2010;16:568-73.

4. Williams K, Scheimann A, Sutton V, et al. Sleepiness and sleep disordered breathing in Prader-Willi syndrome: relationship to genotype, growth hormone therapy, and body composition. J Clin Sleep Med 2008;4:111-18.

5. Brown KA, Samuel S, Patel DR. Pharmacologic management of attention deficit hyperactivity disorder in children and adolescents: a review for practitioners. Transl Pediatr 2018;7:36-47.

6. Tauber M, Diene G, Molinas C, et al. Review of 64 cases of death in children with Prader-Willi syndrome (PWS). Am J Med Genet A 2008;146A:881-7.

7. Angulo MA, Butler MG, Cataletto ME. Prader-Willi syndrome: a review of clinical, genetic, and endocrine findings. J Endocrinol Invest 2015;38:1249-63.

8. Harris RM, Stafford D. Prader Willi syndrome: endocrine updates and new medical therapies. Curr Opin Endocrinol Diabetes Obes 2020;27:56-62.

9. Shephard RJ. Pediatric nutrition in practice. Koletzko B, editor. Basel: Karger Publications, 2008.

10. Beck SE, Marcus CL. Pediatric polysomnography. Sleep Med Clin 2009;4:393-6.

11. Lu W, Qi Y, Cui B, et al. Clinical and genetic features of Prader-Willi syndrome in China. Eur J Pediatr 2014;173:81-6.

12. Kennedy L, Bittel DC, Kibiryeva N, et al. Circulating adiponectin levels, body composition and obesity-related variables in Prader-Willi syndrome: comparison with obese subjects. Int J Obes (Lond) 2006;30:382-87.

13. Sode-Carlsen R, Farholt S, Rabben KF, et al. Body composition, endocrine and metabolic profiles in adults with Prader-Willi syndrome. Growth Horm IGF Res 2010;20:179-84.

14. Höybye C. Endocrine and metabolic aspects of adult Prader-Willi syndrome with special emphasis on the effect of growth hormone treatment. Growth Horm IGF Res 2004;14:1-15.

15. Talebizadeh, Z, Butler MG. Insulin resistance and obesityrelated factors in Prader-Willi syndrome: comparison with obese subjects. Clin Genet 2005;67:230-39.

16. Juul A, Dalgaard P, Blum WF, et al. Serum levels of insulin-like growth factor (IGF)-binding protein-3 (IGFBP-3) in healthy infants, children, and adolescents: the relation to IGF-I, IGF-II, IGFBP-1, IGFBP-2, age, 
sex, body mass index, and pubertal maturation. J Clin Endocrinol Metab 1995;80:2534-42.

17. Tauber M, Barbeau C, Jouret B, et al. Auxological and endocrine evolution of 28 children with Prader-Willi syndrome: effect of GH therapy in 14 children. Horm Res 2000;53:279-87.

18. Butler MG, Theodoro M, Skouse JD. Thyroid function studies in Prader-Willi syndrome. Am J Med Genet A 2007;143A:488-92.

19. Vaiani E, Herzovich V, Chaler E, et al. Thyroid axis dysfunction in patients with Prader-Willi syndrome during the first 2 years of life. Clin Endocrinol (Oxf) 2010;73:546-50.

20. Cassidy SB, Driscoll DJ. Prader-Willi syndrome. Eur J Hum Genet 2009;17:3-13.

21. de Onis M, Onyango AW, Borghi E, et al. Development of a WHO growth reference for school-aged children and adolescents. Bull World Health Organ 2007;85:660-67.

22. Ehara H, Ohno K, Takeshita K. Frequency of the PraderWilli syndrome in the San-in district, Japan. Brain Dev 1995;17:324-26.

23. Kim HJ, Cho HJ, Jin DK, et al. Genetic basis of PraderWilli syndrome in Korea: less uniparental disomy than has been recognized? Clin Genet 2004;66:368-72.

24. Lin HY, Lin SP, Chuang CK, et al. Genotype and phenotype in patients with Prader-Willi syndrome in Taiwan. Acta Paediatr 2007;96:902-5.

25. Coupaye M, Tauber M, Cuisset L, et al. Effect of Genotype and Previous GH Treatment on Adiposity in Adults With Prader-Willi Syndrome. J Clin Endocrinol Metab 2016;101:4895-3.

26. Pavone M, Caldarelli V, Khirani S, et al. Sleep disordered breathing in patients with Prader-Willi syndrome: A multicenter study. Pediatr Pulmonol 2015;50:1354-59.

27. Harris JC, Allen RP. Is excessive daytime sleepiness characteristic of Prader-Willi syndrome? The effects of weight change. Arch Pediatr Adolesc Med 1996;150:1288-93.

28. Butler JV, Whittington JE, Holland AJ, et al. Prevalence

Cite this article as: Lu A, Luo F, Sun C, Zhang X, Wang L, $\mathrm{Lu}$ W. Sleep-disordered breathing and genetic findings in children with Prader-Willi syndrome in China. Ann Transl Med 2020;8(16):989. doi: 10.21037/atm-20-4475 of, and risk factors for, physical ill-health in people with Prader-Willi syndrome: a population-based study. Dev Med Child Neurol 2002;44 :248-55.

29. Sedky K, Bennett DS, Pumariega A. Prader Willi syndrome and obstructive sleep apnea: co-occurrence in the pediatric population. J Clin Sleep Med 2014;10:403-9.

30. Richards A, Quaghebeur G, Clift S, et al. The upper airway and sleep apnoea in the Prader-Willi syndrome. Clin Otolaryngol Allied Sci 1994;19:193-97.

31. O'Donoghue FJ, Camfferman D, Kennedy JD, et al. Sleep-disordered breathing in Prader-Willi syndrome and its association with neurobehavioral abnormalities. J Pediatr 2005;147:823-29.

32. Barbera J, Voloh I, Glenn B, et al. editors. Sleep and Mental Illness. Cambridge: Cambridge University Press, 2010.

33. Urquhart DS, Gulliver T, Williams G, et al. Central sleepdisordered breathing and the effects of oxygen therapy in infants with Prader-Willi syndrome. Arch Dis Child 2013;98:592-95.

34. Cohen M, Hamilton J, Narang I. Clinically important agerelated differences in sleep related disordered breathing in infants and children with Prader-Willi Syndrome. PLoS One 2014;9:e101012.

35. Gallego J. Genetic diseases: congenital central hypoventilation, Rett, and Prader-Willi syndromes. Compr Physiol 2012;2:2255-79.

36. Lindgren AC, Hellstrom LG, Ritzen EM, et al. Growth hormone treatment increases $\mathrm{CO}(2)$ response, ventilation and central inspiratory drive in children with Prader-Willi syndrome. Eur J Pediatr 1999;158:936-40.

37. Haqq AM, Stadler DD, Jackson RH, et al. Effects of growth hormone on pulmonary function, sleep quality, behavior, cognition, growth velocity, body composition, and resting energy expenditure in Prader-Willi syndrome. J Clin Endocrinol Metab 2003;88:2206-12.

(English Language Editor: J. Gray) 